\title{
Bangladesh: COVID-19 Knowledge, Attitudes, Practices and Needs-Responses from the first round of data collection among adolescent girls in five districts
}

Population Council

Follow this and additional works at: https://knowledgecommons.popcouncil.org/departments_sbsr-pgy

Part of the International Public Health Commons, and the Social and Behavioral Sciences Commons How does access to this work benefit you? Let us know!

\section{Recommended Citation}

Population Council. 2020. "Bangladesh: COVID-19 Knowledge, Attitudes, Practices and Needs-Responses from the first round of data collection among adolescent girls in five districts," brief. Dhaka: Population Council. 


\title{
Bangladesh: COVID-19 Knowledge, Attitudes, Practices \& Needs
}

\author{
Responses from the first round of data collection among adolescent girls \\ in five districts
}

April 21-30, 2020

\section{Highlights}

- Over $90 \%$ of adolescent girls correctly reported at least one common symptom; over $80 \%$ correctly identified that proximity to an infected person is a mode of contagion and that social isolation is a recommended preventive practice for COVID-19.

- Social distancing practices are not widely implemented, despite widespread awareness about the importance of avoiding contact with possibly exposed individuals, because of difficulties in distancing during daily activities.

- The lockdown policy is already having a clear economic impact. A high proportion of respondents reported food and resource scarcity and income loss.

- Many adolescent girls reported an increase in care-burden and household work and experienced the psychosocial impact of stress and isolation.

- Girls are adapting to remote learning. One in four girls are participating in a digital classroom initiative telecasted by the Government of Bangladesh.

\section{Bangladesh Context: COVID-19}

The first COVID-19 positive patient in Bangladesh was reported on March 8, 2020. The advent of COVID-19 triggered the closure of all educational institutions. A nationwide "holiday" was declared on March 26, 2020, and a ban was implemented on all passenger travel by water, rail, and air. These restrictions have been partially relaxed, but the disease is spreading rapidly with 28,511 positive cases and 408 deaths as of May 21,2020 . Schools are likely to remain closed through summer.

\section{Rapid Survey on Adolescent Girls}

From April 21-30, the Population Council Bangladesh conducted the first round of a series of rapid phone-based surveys on COVID-19 knowledge, attitudes, and practices (KAP) and the social and economic impacts of COVID-19 on adolescent girls. While there are a number of rapid surveys underway to assess the impact of COVID-19 in the country, relatively little is known about its impact on young people. Protracted school closures may increase the risk of school dropout, which would result in wide-ranging lifelong consequences for adolescents. With $59 \%$ of women marrying before the age of 18, Bangladesh has the highest prevalence of child marriage in Asia; this may increase with COVID-19. Through repeated surveys, we plan to assess the impact on girls' vulnerability to school dropout and explore program efforts that may contribute to protecting girls. 
The survey target was to sample 1,000 girls from program areas of two ongoing projects to end child marriage in five rural districts. Fifty percent were randomly drawn from a list of girls enrolled in programs, and $50 \%$ were drawn from a representative sample of all girls.

In total, 1,416 girls had to be contacted to successfully complete 959 interviews yielding a $68 \%$ contact success rate. The most common reason for failure to complete was a wrongly recorded or incomplete phone number. Comparison with in-person surveys from the same populations suggests the phone survey sample is representative and unbiased with no statistically significant difference in overall sample characteristics such as age, marital status, and schooling. The average age of respondents was 15 years. Eight in 10 respondents were currently in school and had at least some secondary education. Twelve percent were married and $8 \%$ reported involvement in some incomegenerating activities.

\section{Knowledge on Infection, Symptoms, and Prevention Measures}

While general awareness about symptoms, contagion and prevention was unexpectedly high, the data also reflect the evolving nature of health advisory in the "info-demic" associated with COVID-19.

- Fever (94\%) and dry cough (72\%) were correctly identified while headache and muscle pain (44\%) and difficulty breathing as a severe symptom (43\%) were less well-known.

- Some symptoms were commonly misidentified. The misidentification of

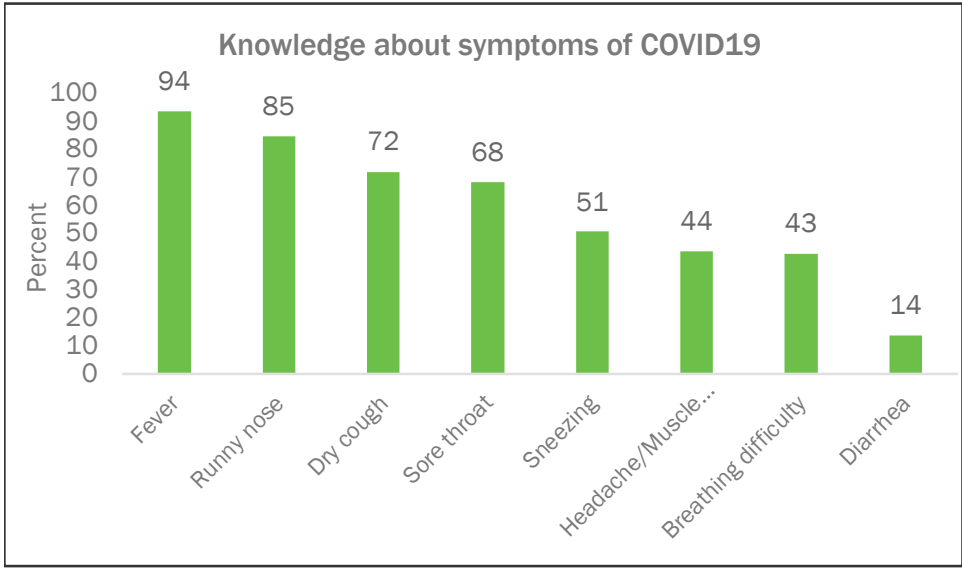
runny nose as a symptom may be because a colloquial expression "shordi" encompasses stuffy nose, mucus, and runny nose. A widely publicized campaign about coughing and sneezing into one's elbow possibly contributed to identifying sneezing as a symptom.

- With regards to transmission, respondents spontaneously identified infected persons (81\%) and coughing/sneezing droplets from infected people (77\%) as modes of contagion of the coronavirus. Only $29 \%$ of the respondents know that the virus can be spread by touching a contaminated surface or object. Thirtytwo percent of respondents know that asymptomatic people can spread COVID-19.

- Respondents were largely aware of prevention behaviors. Knowledge of staying home (82\%), washing hands (84\%), and use of mask (74\%) were very common across age and education levels.

- Specific knowledge, such as covering mouth while coughing and sneezing (41\%), practicing physical distancing when outside (53\%), avoiding contact with people suffering from fever and cough (29\%), and having plenty of foods high in vitamin C to boost immunity (14\%), were less frequently identified.

- With regard to actions, if presenting symptoms, knowledge of self-isolation at home (68\%) and going to the hospital in case of severe symptoms (51\%) was common while calling a helpline or hotline number for telemedicine and advice was comparatively low (35\%).

\section{Trusted Sources of Information}

When asked about what they considered trusted sources of information, 90\% reported newspapers, radio, or TV; $61 \%$ identified family, friends and relatives; $35 \%$ cited awareness-raising activities in the community; and 32\% relied on social media (Facebook, YouTube, Twitter, etc.) and $23 \%$ on calls/SMS. 


\section{Prevention Practices}

Increased handwashing and use of face masks were reported by most of the adolescent girls as preventive practices, while maintaining social distancing was not commonly reported as a personal behavior change since the onset of the pandemic.

- Seven in 10 girls said they wore face masks outside in the past week.

- Seventy-one percent of the adolescent girls washed hands more frequently than the previous weeks. They also reported staying home more, declining to meet with friends, withholding private tutoring, and checking news more frequently.

\section{Social Distancing}

Thirty-five percent of adolescent girls reported that they had gone outside in the last 24 hours, and $70 \%$ reported that a member of her family went outside

in the last 24 hours during the lockdown period. While girls reported staying at home for an average length of seven days, they reported that family members only stayed home for one day. These data reflect that lockdown practices are not rigid and allow for mobility. Shops and bazaars were open with limited hours, and some essential services were available.

\section{Effects on Daily Life}

COVID-19 is having a significant impact on the education, health, economic, and social lives of the adolescents and their families.

\section{Household Income and Food Security}

The negative economic impact was evident with girls reporting food scarcity and income loss in their families. Half of the respondents said their household earned no income at all during lockdown and one-fifth reported an urgent need for food.

\section{Girls' Education}

Nine in 10 adolescent girls are continuing their study at home by themselves and/or with a household member's help. Twenty-six percent of adolescent girls are following lessons broadcast on national television. Only $7 \%$ of girls reported that they are not studying at all during this lockdown.

\section{Care Burden}

Adolescent girls are often relied on for caretaking, and these responsibilities may be increasing under lockdown.

- Increased care responsibilities for ill family members have been reported by adolescent girls. In addition, $22 \%$ of adolescent girls reported an increase in household chores during lockdown because migrant household members returned home due to workplace closures.

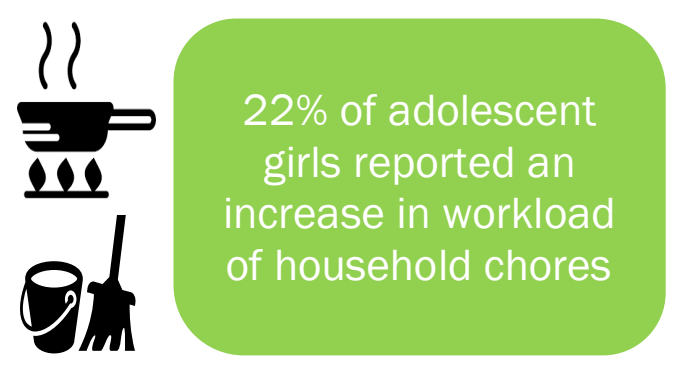




\section{Mental health, abuse, and violence}

- Seventy-five percent reported feeling sometimes or mostly depressed during the lockdown period.

- Eighteen percent reported that they faced some form of violence (rebuke, scolding, beating) in the last two weeks. Also, $28 \%$ of the adolescent girls reported increased cases of violence in their surroundings in the last two weeks. Reports of violence were higher among older adolescent girls and married girls compared to their unmarried and younger adolescent girls.

\section{Immediate Needs of Families}

Respondents listed the most essential things they and their families need during the lockdown period. Food, soap, and medicine were reported as the major items needed.

\section{Subgroup}

\section{Considerations}

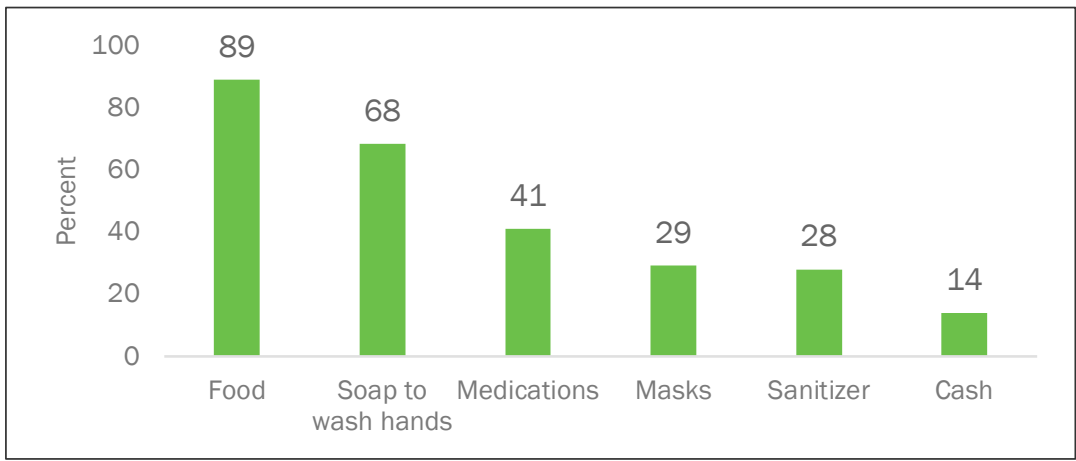

Girls age 15 and below reported less accurate knowledge of transmission, symptoms, and preventive behaviors compared to girls age 16 and above. Older adolescents were more likely to engage in increased household work (31\% versus $18 \%$ ) and care-taking responsibilities compared to the younger adolescent group (63\% versus $46 \%$ ).

Married adolescents reported more household violence than their unmarried peers (35\% versus 16\%) and a higher percentage of married adolescents reported constant depression compared to their unmarried peers during lockdown (23\% versus 14\%).

\section{Recommendations}

The Government of Bangladesh should strengthen its public education campaigns and provide clear messages on symptoms of COVID-19, including information accessible to younger age groups.

- Communicate clear messaging about severe COVID-19 symptoms, such as difficulty breathing, through mass media. Television and radio were reported to be the most trusted and accessed.

- Promote the practice of social distancing heavily as well as help-seeking such as calling a hotline for advice and staying home/self-isolating if one has mild symptoms.

- In the education system, increase awareness with messaging in the life skills curriculum as younger adolescent girls (ages 10-15) seem to have comparatively less knowledge about COVID-19. Girls were generally not aware of the possibility of asymptomatic spread, so this information should be a focus of the communication efforts.

- Integrate COVID-19 messaging into government-led classes broadcasted on television. One-fourth of adolescent girls are taking up and following these classes.

Government efforts should also address the effects of income loss and food insecurity. Provide direct assistance to girls and women to help them cope with the challenges of increased household and care work.

- Provide access to mental health services remotely and protection for violence as adolescent girls reported increased incidents of depression and violence.

Partnering with national health ministries and other government agencies in sub-Saharan Africa, South Asia, and Latin America, Population Council global and in-country scientists are conducting COVID-19 public health and social science research to produce relevant and timely evidence to support policymakers in controlling the spread of coronavirus, evaluating the effectiveness of prevention and mitigation measures, and assessing longer-term health, social and economic effects of the pandemic.

Suggested citation: Population Council. 2020. "Bangladesh: COVID-19 Knowledge, Attitudes, Practices \& Needs: Responses from the first round of data collection among adolescent girls in five districts." 